Received: 2017.12 .20

Accepted: 2018.02.25

Published: 2018.06 .28

Authors' Contribution:

Study Design A

Data Collection B

Statistical Analysis C

Data Interpretation D

Manuscript Preparation E

Literature Search F

Funds Collection G

\title{
Ouabain Protects Mice Against Lipopolysaccharide-Induced Acute Lung Injury
}

\author{
BCE 1 Changli Wang* \\ BCE 1 Yan Meng* \\ CF 1,2 Yuanyuan Wang \\ DF 1 Zhengyu Jiang \\ C 1 Mengda Xu \\ AE 1 Lulong Bo \\ AG 1 Xiaoming Deng
}

1 Faculty of Anesthesiology, Changhai Hospital, Naval Medical University, Shanghai, P.R.China

2 Department of Anesthesiology, Women and Children's Health Care Hospital of Linyi City, Linyi, Shandong, P.R. China
Corresponding Authors:

Source of support:

* Changli Wang and Yan Meng contributed equally to this work

Xiaoming Deng, e-mail: deng_x@yahoo.com, Lulong Bo, e-mail: nbastars@126.com

This work was supported by the National Natural Science Foundation of China (No. 81471845; 81671887) and Shanghai Outstanding Youth Medical Professionals Training Program (2017YQ015)

Background:

Material/Methods:

Results:

Conclusions:

MeSH Keywords:

Full-text PDF:
Ouabain, an inhibitor of $\mathrm{Na}^{+} / \mathrm{K}^{+}-$ATPase, is a type of endogenous hormone synthesized in the adrenal cortex and hypothalamus. Previous studies found that ouabain potently inhibited inflammatory reactions and regulated immunological processes. Our present study aimed to investigate the therapeutic role of ouabain on lipopolysaccharide (LPS)-induced acute lung injury (ALI) in mice.

Ouabain $(0.1 \mathrm{mg} / \mathrm{kg})$ or vehicles were intraperitoneally injected into male C57BL/6J mice once a day for 3 consecutive days. One hour after the last injection of ouabain, LPS $(5 \mathrm{mg} / \mathrm{kg})$ was administrated through intranasal instillation to induce ALI. 6 hours and 24 hours later, bronchoalveolar lavage fluid (BALF) and lung tissues were harvested to detect the protective effects of ouabain, including protein concentration, inflammation cell counts, lung wet-to-dry ratio, and lung damage.

The results showed that ouabain attenuated LPS-induced ALI in mice, which was indicated by alleviated pathological changes, downregulated TNF- $\alpha$, IL-1 $\beta$, and IL- 6 production, inhibited neutrophils infiltration and macrophages, and ameliorated pulmonary edema and permeability. Further results found the activation of nuclear factor-kappa B (NF-kB) and mitogen-activated protein kinase (MAPK) signaling pathways were suppressed by ouabain in LPS-induced ALI.

These results suggest that ouabain negatively modulates the severity of LPS-induced ALI.

Acute Lung Injury • Lipopolysaccharides • Mitogen-Activated Protein Kinase Kinases • NF-kappa B

https://www.medscimonit.com/abstract/index/idArt/908627

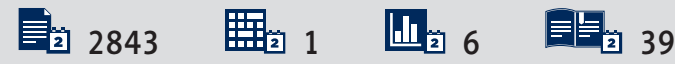




\section{Background}

Acute lung injury (ALI) is a kind of clinical syndrome of acute respiratory failure that usually caused by sepsis, pneumonia, or severe trauma, which has an extensive influence on health [1]. The pathological characteristics of ALI are the increased permeability of the alveolar-capillary barrier, neutrophils accumulation, uncontrolled inflammation, and oxidative stress injury [2]. Despite extensive investigations on early diagnosis and therapy of ALI, the incidence and mortality of this disease still remain high in patients [2]. Therefore, effective therapeutic options towards ALI are urgently needed.

Ouabain is a kind of $\mathrm{Na}^{+} / \mathrm{K}^{+}$-ATPase inhibitor, which has been used in the therapy of congestive heart failure therapy for a long time. It is also an endogenous substance synthesized in the mammals' adrenal cortex and hypothalamus, which plays an important role in physiological regulations [3,4]. Research has shown that the ouabain level in serum was increased with the stimulation of blood volume augmentation, angiotensin II, adrenocorticotropic hormone, hypertension, or stress conditions $[5,6]$. Compelling evidence has demonstrated that ouabain regulates immune functions. It inhibits phosphorylated MAPK p38 expression in concanavin A activated monocytes [7]. In addition, ouabain regulates the process of inflammation by inhibiting TNF- $\alpha / N F-\kappa B$ signaling pathway in HeLa cells and $293 T$ cells [8]. Recent data demonstrated the anti-inflammatory and analgesic effects of ouabain by inhibiting NF- $\mathrm{KB}$ activation $[9,10]$. It has also been demonstrated that ouabain could negatively modulate airway inflammation in ovalbumin-induced asthma [11]. However, whether ouabain could regulate inflammatory responses in LPS-induced ALI in mice has not been reported.

Using a well-established model of ALI, through intranasal instillation LPS, we aimed to investigate whether ouabain reduces LPS-induced ALI. We report herein that ouabain ameliorates LPS-induced lung histopathologic changes, reduces inflammatory cells infiltration, and attenuates the severity of lung injury in mice.

\section{Material and Methods}

\section{Reagents}

LPS, Evans blue dye, and ouabain were purchased from Sigma Chemical Co (St Louis, MO, USA). Mouse TNF- $\alpha$, IL-1 $\beta$, and IL-6 enzyme-linked immunosorbent assay (ELISA) kits were purchased from R\&D system (Minneapolis, MN, USA). Mouse p65, p-p65, p38, p-p38, ERK, p-ERK, JNK, and p-JNK were provided by Cell Signaling Technology Inc (Beverly, MA, USA). The fluorescent-labeled monoclonal antibodies anti-F4/80-FITC,
anti-CD11b-APC, anti-Ly6G-PE were bought from eBiosciences (San Jose, CA, USA).

\section{Animals}

Health 6- to 8-week-old male C57BL/6J mice were purchased from Shanghai SLAC Laboratory Animals Center (Shanghai, China). All mice were housed at a 12-hour light-dark cycle environment, with free access to water and food. Animal experiments were approved by the Scientific Investigation Board of Naval Medical University.

\section{Administration of ouabain}

According to previous research [10-12], in our preliminary experiments, we also tried to evaluate whether the effect of ouabain was in a dose-dependent manner in a mouse model of ALI. We adopted $0.02,0.1$, and $0.5 \mathrm{mg} / \mathrm{kg}$ of ouabain intraperitoneal injection into mice within 3 consecutive days. Our results showed that the dose of $0.1 \mathrm{mg} / \mathrm{kg}$ was much more effective to inhibit the inflammatory responses in vivo (Supplementary Figure 1). We also found that ouabain treatment after LPS challenge was less effective than ouabain pretreatment method (Supplementary Figure 2). Thus, we chose the dose of $0.1 \mathrm{mg} / \mathrm{kg}$ ouabain and the administered within 3 consecutive days before LPS challenge in our research to explore its effectiveness.

\section{Animal models of ALI}

C57BL/6J mice were randomly assigned to the following groups ( $n=8$, each): 1) control group; 2) LPS treated group; 3) LPS and ouabain treated group. Each mouse was intraperitoneally injected the same volume $(200 \mu \mathrm{L})$ of vehicles (PBS) or ouabain $(0.1 \mathrm{mg} / \mathrm{kg})$ once a day for 3 consecutive days. One hour after the last injection of ouabain, animals were anesthetized by sevoflurane, and LPS ( $5 \mathrm{mg} / \mathrm{kg}$ ) dissolved in $50 \mu \mathrm{L}$ PBS was then administered through intranasal instillation to induce ALI; the mice in the control group received equivalent PBS [13]. At 6 hours and 24 hours after LPS challenge, all mice were sacrificed by $\mathrm{CO}_{2}$ inhalation. Then, lung tissues and bronchoalveolar lavage fluid (BALF) were collected.

\section{Histology}

Lungs were fixed in $4 \%$ paraformaldehyde solution for more than 48 hours before being embedded in paraffin and sectioned. After deparaffinization and rehydration, the sections were stained with hematoxylin and eosin (H\&E). Alveolar congestion, hemorrhage, aggregation of inflammatory cells, and the thickness of the alveolar walls were assessed under a light microscope [14]. 


\section{Lung W/D weight ratio}

The lung tissues in each group were harvested, and the wet weights were measured. Then the lungs were incubated in an oven at $80^{\circ} \mathrm{C}$ for 3 days to remove moisture, and the dry weights were measured. The wet to dry weight ratio was calculated to assess the edema.

\section{Evans blue staining in lungs}

Evans blue dye accumulation in lungs was measured [15]. Mice were administered $25 \mathrm{mg} / \mathrm{kg}$ Evans blue dye by tail vein injection 2 hours before lung tissues were harvested. Before excised, lungs were perfused with PBS containing heparin to remove blood. Then, Evans blue dye in lung tissues was extracted and quantitated by spectrophotometric method.

\section{Bronchoalveolar lavage fluid analysis}

The BALF was obtained by intratracheal injection with $1 \mathrm{~mL}$ cold PBS. Then the BALF was centrifuged at $1500 \mathrm{rpm}$ for 10 min. The cells in BALF were collected and stained with antiF4/80-FITC and anti-CD11b-APC to detect macrophages, stained with anti-Ly6G-PE and anti-CD11b-APC to detect neutrophils by flow cytometry (BD Bioscience, San Jose, CA, USA) [15]. Flow cytometry data was acquired using FACSCanto II flow cytometer (BD Bioscience, San Jose, CA, USA) and analyzed using FlowJo software, version 7.6.1 (Tree Star, Ashland, OR, USA). The total proteins in the supernatant were measured by BCA method (Thermo scientific, IL, USA). For cytokines detection, TNF- $\alpha$, IL-1 $\beta$, and IL- 6 levels in BALF were measured by ELISA with commercial kits.

\section{Blood cell counts analysis}

Mice were anesthetized by sevoflurane 6 hours and 24 hours after LPS challenge. Blood was collected by heart puncture. Then, the blood was sent into ethylenediaminetetraacetic acid tube and detected by MINDRAY animal automatic blood cell analyzer (Model: BC-2800vet).

\section{Real-time PCR}

Total RNA in lung tissues were isolated using the TRIzol reagent (Takara Biotechnology, Dalian, China) based on the instructions. Reverse-transcribed into cDNA was performed using a PrimeScript RT reagent kit (Takara Biotechnology, Dalian, China). Determined and normalized by the amount of B2M, the $2-\Delta \Delta C t$ method was used to calculate the relative gene expression. The murine primers were synthesized in Invitrogen as follows:

TNF- $\alpha$ : F 5'-CACCATGAGCACAGAAAGCA-3'

R 5'-TAGACAGAAGAGCGTGGTGG-3'
IL-1ß: F 5'-ACTCATTGTGGCTGTGGAGA-3'

R 5'-TTGTTCATCTCGGAGCCTGT-3'

IL-6: F 5'-ACCACTCCCAACAGACCTG-3'

R 5'-GGTACTCCAGAAGACCAGAGG-3'

B2M: F'-CGGCCTGTATGCTATCCAGA-3'

R 5'-GGGTGAATTCAGTGTGAGCC-3'

\section{Western blot analysis}

Immunoblotting was performed according to standard methods. Briefly, equal amounts of proteins in lung tissues were separated on SDS-polyacrylamide gel electrophoresis, transferred to PVDF membranes and subsequently incubated with antibodies: p65, p-p65, p38, p-p38, ERK, p-ERK, JNK, and p-JNK (Cell Signal Technology, Beverly, MA, USA). Membranes were then incubated with the peroxidase-conjugated secondary antibodies. At last, the signals were visualized by enhanced chemiluminescence (Pierce, Rockford, IL, USA). The relative band intensity was quantified using ImageJ 1.47v (Rawak Software, Inc. Germany).

\section{Statistical analysis}

Statistical analysis was conducted with GraphPad Prism 5 (GraphPad Software Inc., CA, USA) using one-way analysis of variance (ANOVA) and the 2-tailed Student's $t$-test. All data were presented as mean values \pm SEM. $P$ values $<0.05$ were considered statistically significant.

\section{Results}

\section{Ouabain reduces LPS-induced lung histopathologic changes}

In the present study, at 6 hours and 24 hours after LPS challenge, lungs from mice of each group were collected and stained with $\mathrm{H} \& \mathrm{E}$ to determine the effect of ouabain on LPS intranasal instillation induced ALI. As shown in Figure 1A, interstitial edema, alveolar hemorrhage, the thickness of pulmonary septum, and inflammatory cells infiltration were obviously observed in LPS group. However, the histopathological changes of lungs were alleviated by ouabain administration. Compared with the control group, the pathological scores increased after LPS challenge, while the pathological scores were reduced by the treatment of ouabain (Figure 1B). Thus, these data demonstrated that ouabain could ameliorate LPS-induced lung injury in mice.

\section{Ouabain ameliorates LPS-induced inflammatory cells infiltration}

In the progression of $\mathrm{ALI}$, the migration of neutrophils is one of the hallmark events. Neutrophils and alveolar macrophages 
A

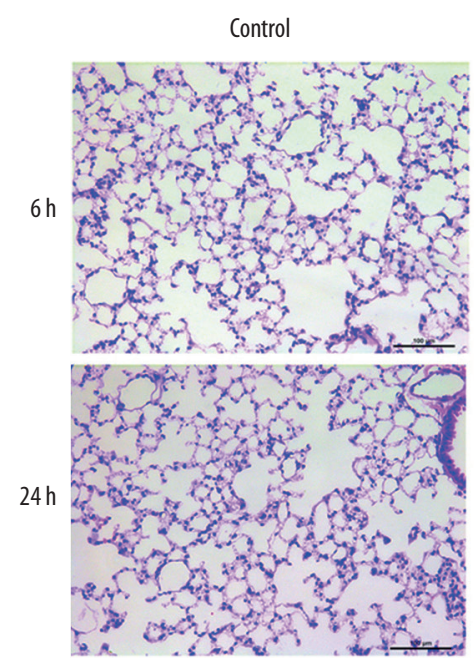

Control
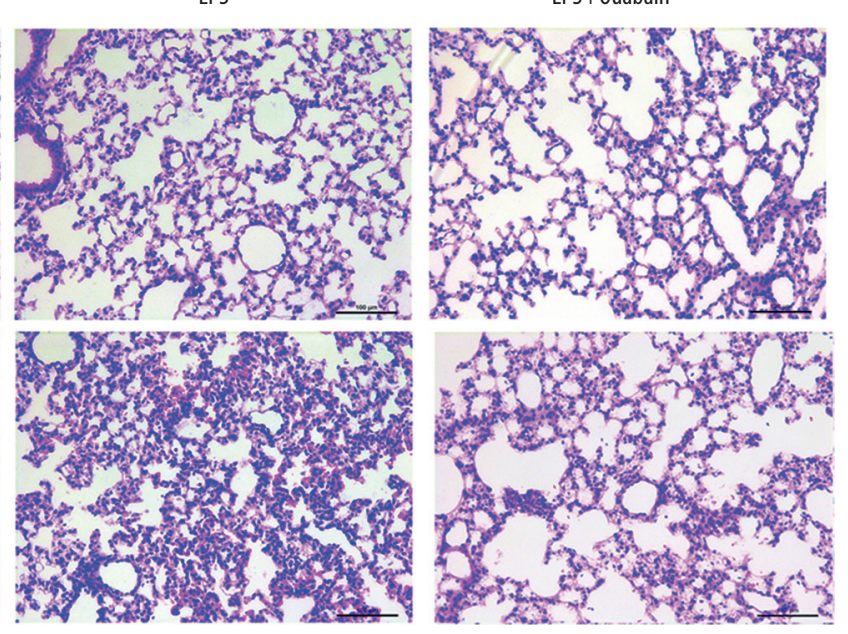

B

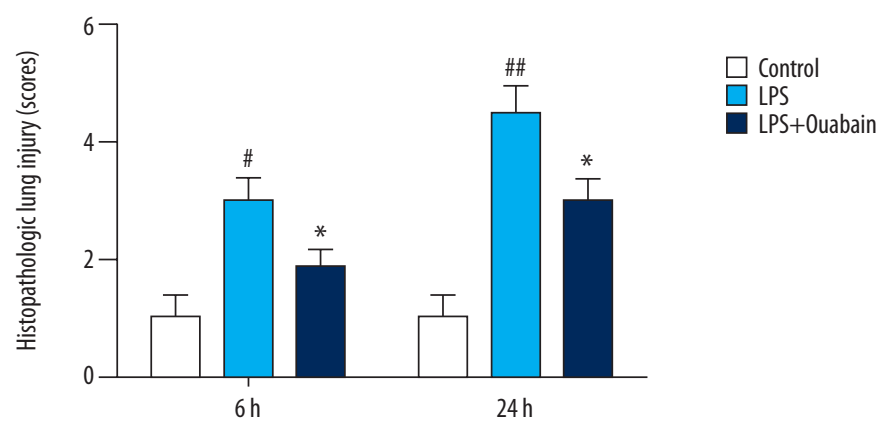

Figure 1. Effects of ouabain on LPS-induced lung histopathologic changes in mice. (A) At 6 hours and 24 hours after LPS challenge, lungs in each group were prepared for histological evaluation. Representative histological section of the lungs was stained by $H \& E$ staining, magnification (200x). (B) The lung injury scores were determined. The values presented are mean $\pm \operatorname{SEM}(n=8)$, ${ }^{\#} P<0.05$, \#\# $P<0.01$ vs. the control group; * $P<0.05$ vs. the LPS-treated group.

are vital to defending against pathogen invasion and infection in ALI [15]. Therefore, we measured the effects of ouabain on inflammatory cells infiltration in the lungs. We found that LPS markedly increased the number of total cells, neutrophils, and alveolar macrophages in BALF at 6 hours and 24 hours after LPS challenge. However, those increases were reduced by the administration of ouabain (Figure 2A-2C). We also examined the blood cell counts in mice of each group. We found that the number of white blood cells and neutrophils significantly increased at 6 hours and 24 hours after LPS challenge. However, there were no significant differences between the ouabain group and LPS group 6 hours and 24 hours after the administration of LPS, respectively (Table 1). In summary, these results indicated that ouabain has an effect on inhibiting the migration of neutrophils and macrophages into the pulmonary alveoli.

\section{Ouabain alleviates the permeability of lungs induced by LPS}

The permeation of macromolecules and fluid into the interstitium was increased when the endothelial cell barrier was damaged [16]. Therefore, we measured the lung W/D ratio, total proteins in BALF, and the effusion of Evans blue dye to reflect the severity of lung permeability and edema. As shown in Figure 2D, the lung W/D ratio was markedly increased after LPS administration 6 hours and 24 hours. However, ouabain significantly decreased the lung W/D ratio. Furthermore, ouabain reduced total proteins in BALF (Figure 2E), which indicated that ouabain could reduce the severity of lung permeability. Evans blue dye was injected into the caudal vein to evaluate the vascular leakage in lungs. Compared with the LPS group, the leakage of Evans blue dye from the vessels into the interstitium in the ouabain treated mice was significantly reduced (Figure 2F), which indicated a protective effect of ouabain on 


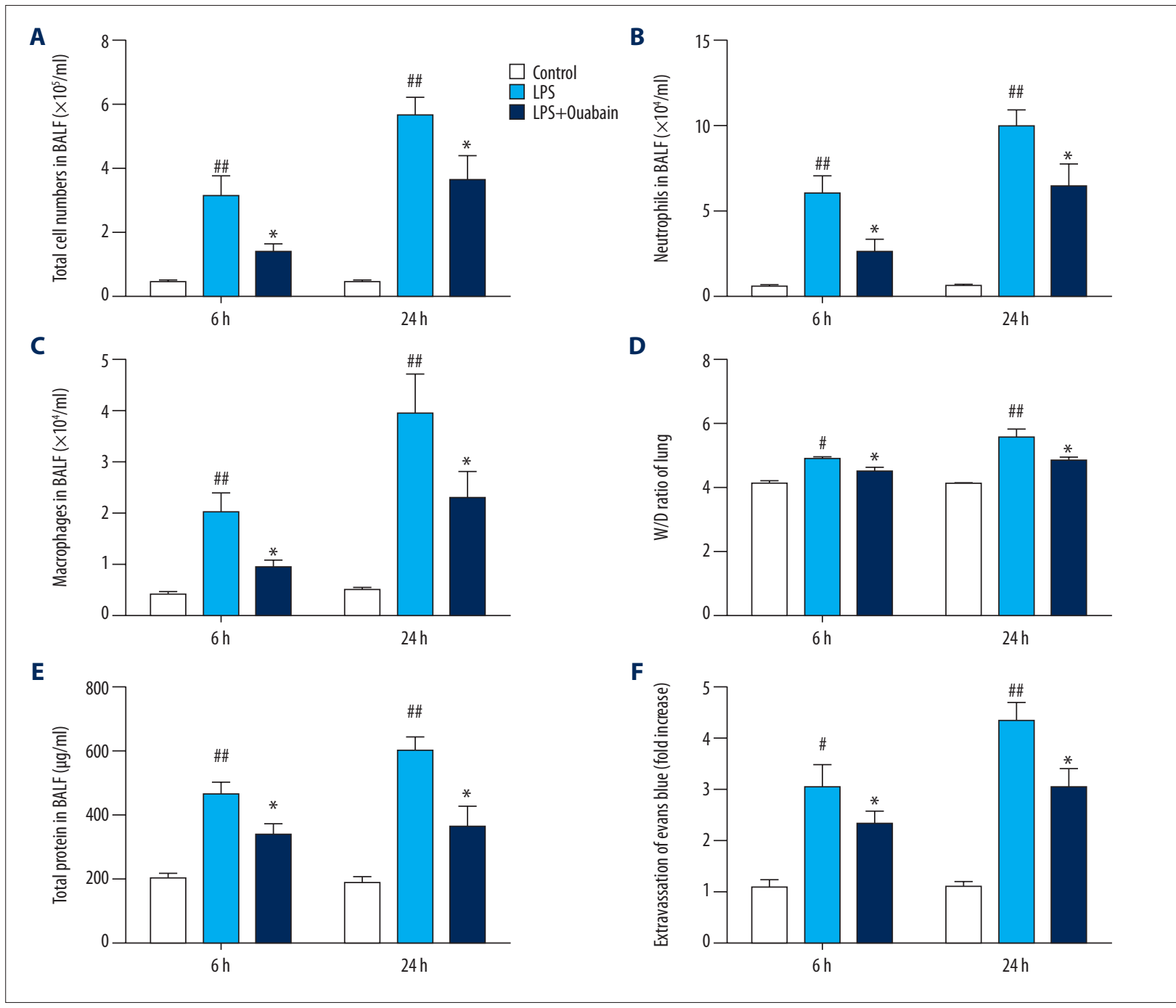

Figure 2. Effects of ouabain on inflammatory cells and permeability of lungs induced by LPS. Lung tissues and BALF were harvested to investigate at 6 hours and 24 hours after LPS treatment. (A-C) The total cells, neutrophils, and alveolar macrophages were detected by flow cytometry in BALF. (D) Lung tissues were weighed and calculated the W/D ratio. (E) BCA method was used to measure the total proteins in BALF. (F) The permeability of lungs was detected by injecting Evans blue dye. The values presented are mean \pm SEM $(\mathrm{n}=8),{ }^{*} P<0.05, \# \#<<0.01$ vs. the control group; ${ }^{*} P<0.05$ vs. the LPS-treated group.

Table 1. The blood cell counts ( $n=8$, each group).

\begin{tabular}{|cccccc} 
& Normal & LPS (6 h) & OUB (6 h) & LPS (24 h) & OUB (24 h) \\
\hline WBC $\left(10^{9} / \mathrm{L}\right)$ & $2.07 \pm 1.26$ & $4.25 \pm 1.07^{*}$ & $4.92 \pm 0.51$ & $5.42 \pm 1.70$ & $5.1 \pm 1.32$ \\
\hline Neutrophil $(10 \% / L)$ & $0.59 \pm 0.27$ & $1.92 \pm 0.52^{*}$ & $2.23 \pm 0.17$ & $2.05 \pm 0.52$ & $2.35 \pm 0.42$ \\
\hline Neutrophil $(\%)$ & $23.35 \pm 5.44$ & $43.27 \pm 2.10^{*}$ & $42.96 \pm 5.12$ & $37.57 \pm 5.51$ & $41.83 \pm 6.54$ \\
\hline
\end{tabular}

* $P<0.05$, compared with normal group. 


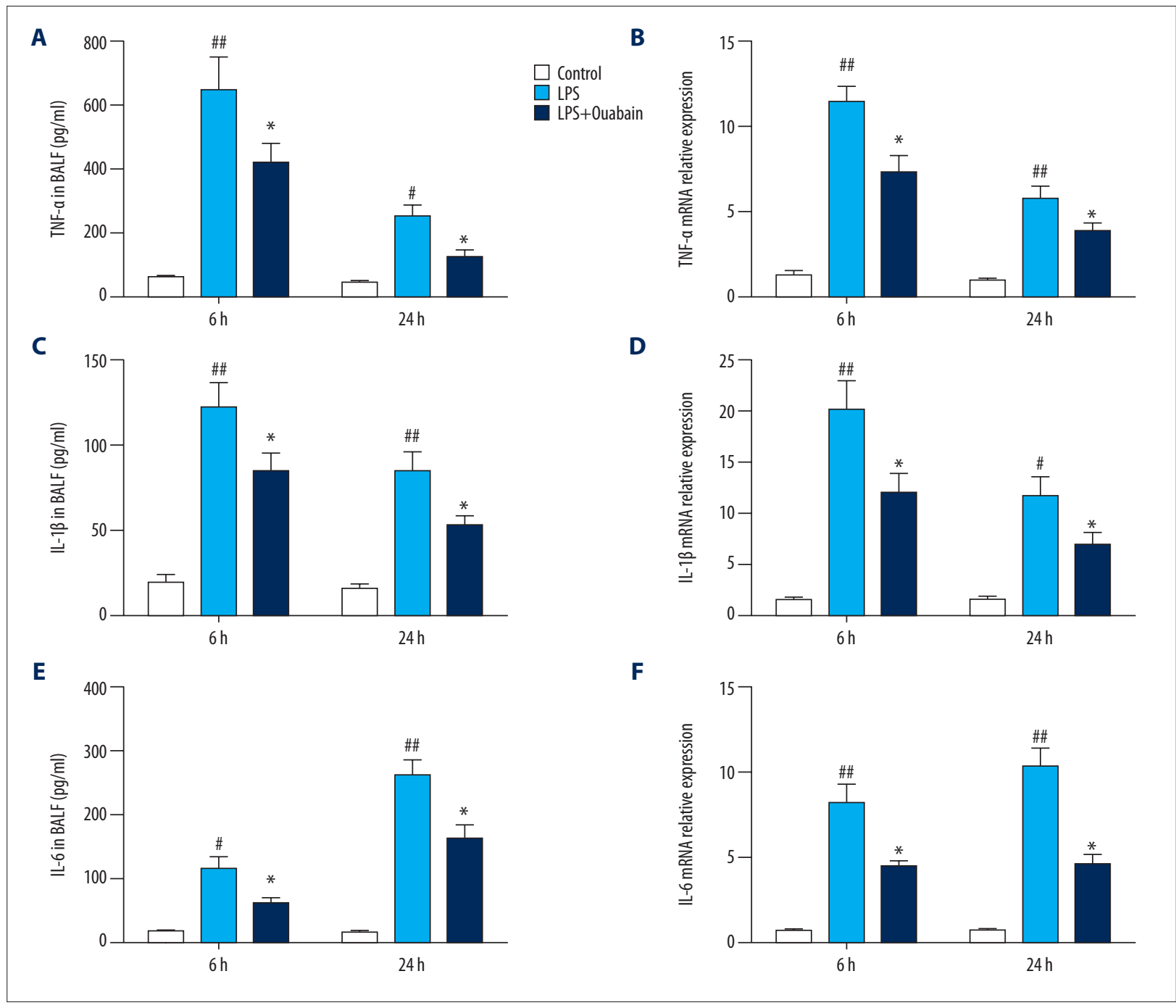

Figure 3. Effects of ouabain on the production of inflammatory cytokines in LPS-induced ALI. (A, C, E) BALF was collected and the levels of TNF- $\alpha$, IL-1 $\beta$, and IL- 6 was measured at 6 hours and 12 hours after LPS challenge. (B, D, F) The mRNA expression of TNF- $\alpha$, IL-1 $\beta$, and IL- 6 were detected in lung tissues. The values presented are mean \pm SEM $(n=8), \# P<0.05, \# \# P<0.01$ vs. the control group; * $P<0.05$ vs. the LPS-treated group.

the endothelial cell barrier in LPS-induced ALI. All above demonstrated that ouabain could alleviate lung edema and permeability in LPS-induced ALI in mice.

\section{Ouabain suppresses LPS-induced lung inflammatory cytokines}

Inflammatory cytokines play a major role in LPS-induced ALI. In order to illustrate whether ouabain could inhibit LPS-induced inflammation in lungs, we measured the levels of TNF- $\alpha$, IL-1 $\beta$, and IL- 6 in BALF. At 6 hours and 24 hours after LPS challenge, compared with the control group, the level of these cytokines was significantly increased (Figure 3A, 3C, 3D), while ouabain suppressed their upregulation. Besides, the gene expression of these inflammatory cytokines in the lungs was similar to the protein level in BALF. In lung homogenates, the gene expression of TNF- $\alpha$, IL- $1 \beta$, and IL- 6 was reduced respectively by the administration of ouabain (Figure 3B, 3D, 3E). Therefore, ouabain is able to suppress inflammatory mediators in LPS-induced ALI in mice.

\section{Ouabain inhibits lung NF- $\kappa B$ and MAPK signaling pathways}

The NF- $\kappa B$ and MAPK signaling pathways are required for LPS mediated inflammatory responses [17]. Therefore, we examined the phosphorylation of NF-KB and MAPK signaling pathways in lung homogenate to explore the molecular evidence of the anti-inflammatory effects of ouabain. As illustrated in Figure 4, the expression of the phosphorylated ERK, JNK, p65, and p38 at the 6 hours and 24 hours after LPS challenge was 


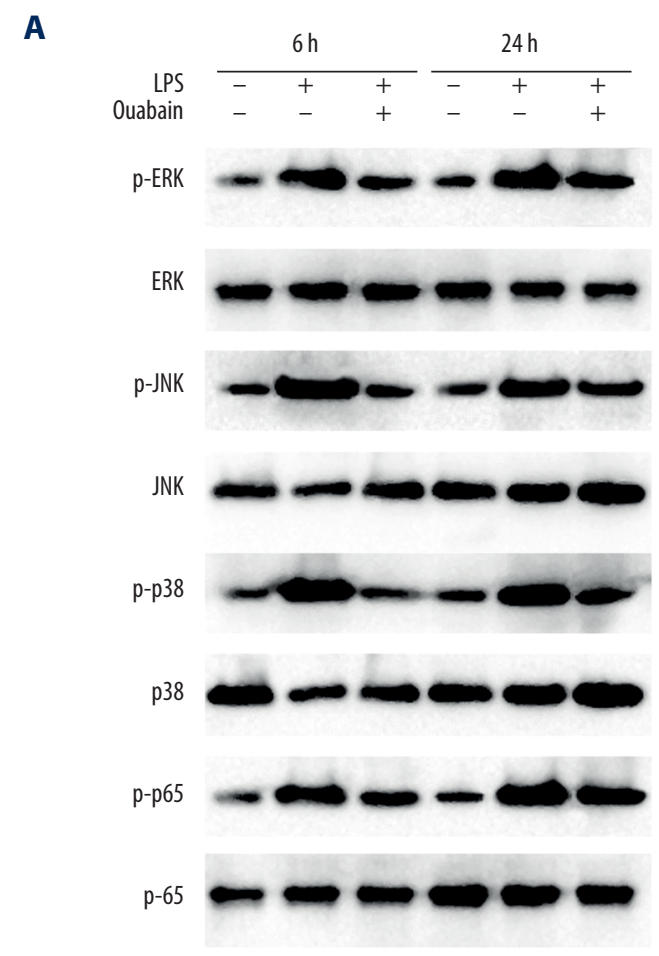

B

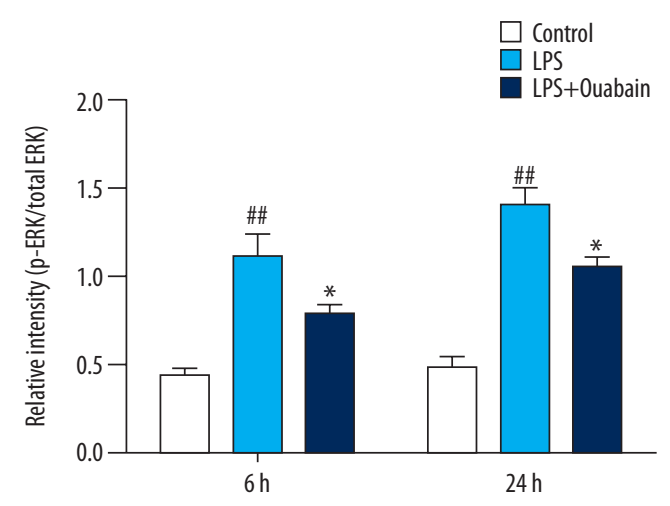

C

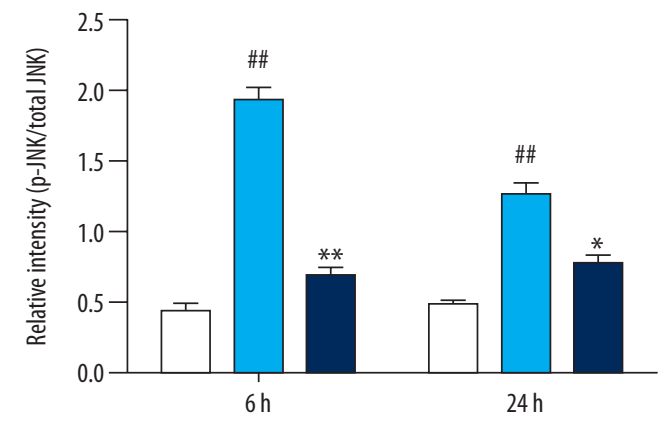

D

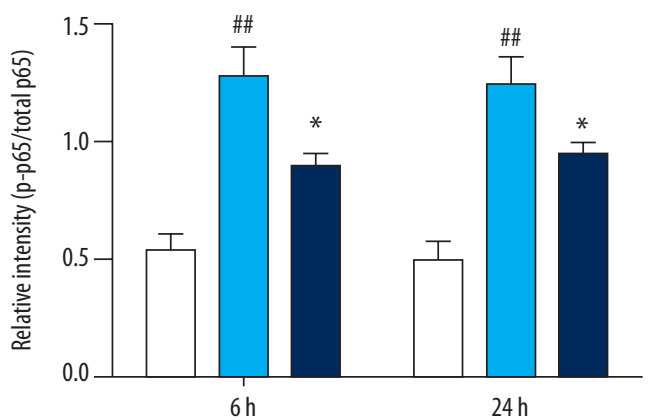

E

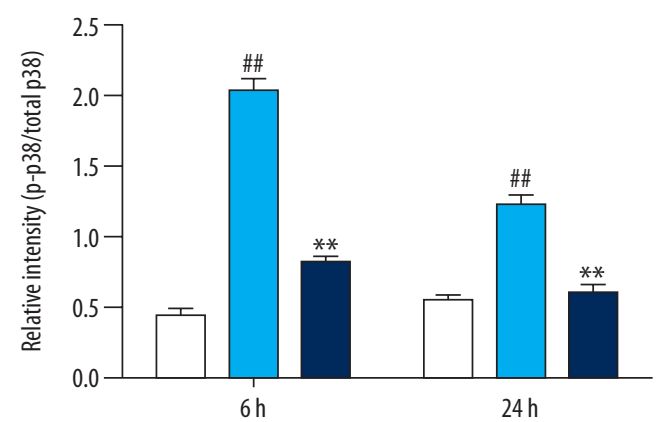

Figure 4. Effects of ouabain on NF-KB and MAPK signaling expression in lungs. (A) Protein levels of p-ERK, ERK, p-JNK, JNK, p-p65, p65, p-p38, and p38 in lung homogenates were evaluated by western blot analysis after LPS challenge 6 hours and 24 hours later. (B-D) Densitometric analysis of the relevant bands was performed. The values presented are mean \pm SEM $(n=3)$. The data are representative of 3 independent experiments. ${ }^{\#} P<0.05$, ${ }^{\#} P<0.01$ vs. the control group; ${ }^{*} P<0.05,{ }^{* *} P<0.01$ vs. the LPS-treated group.

significantly increased. Ouabain inhibited the phosphorylation of these signaling molecules. Our data indicate that ouabain treatment attenuates the severity of LPS-induced ALI.

\section{Discussion}

In this study, we found that ouabain attenuates LPS-induced ALI by inhibiting inflammatory responses. LPS is a component of the cell walls of Gram-negative bacteria, which can induce neutrophils recruitment, inflammation, vascular leakage, and tissues damage [18]. The key pathogenesis of LPS-induced ALI is the production of the inflammatory cells and excessive inflammatory cytokines in lungs [19]. LPS increased the production and the secretion of inflammatory cytokines in ALI, which was relevant to the severity of lung injury [20]. Research has shown that suppressing the release of inflammatory cytokines could reduce the incidence and severity of ALI [21]. Recently, the anti-inflammatory role of ouabain has been widely studied. It reduced TNF- $\alpha$ and IFN- $\gamma$ in acute inflammatory responses induced by Leishmania amazonensis infection [9]. Researchers have demonstrated that ouabain inhibited zymosan-induced 
peritonitis, which was related to the reduction of TNF- $\alpha$ and IL-1 $\beta$ [12]. Teixeira and colleagues found that ouabain enhanced the expression of anti-inflammatory cytokine IL-10 in human monocytes [22]. Low doses of ouabain could reverse the immune paralysis of sepsis by increasing TNF- $\alpha$, IFN- $\gamma$, and GMCSF expression and improving the survival rate of mice [23]. However, studies have shown that ouabain induced lung inflammation and cytokines production, which may be associated with the differences on the dose and route of administration [24]. Although studies have shown that ouabain has a proinflammatory effect, more researches have focused on the inhibitory effect of ouabain on inflammation [25]. Therefore, we adopted LPS-induced ALI in our study to better understand the immunomodulatory effect of ouabain. We found the production of TNF- $\alpha$, IL-1 $\beta$, and IL- 6 in LPS-induced ALI was significantly inhibited by the administration of ouabain in vivo, which illustrated that ouabain could suppress cytokines production to inhibit the inflammatory responses in LPS-induced ALI in mice.

Although infiltrated neutrophils can engulf bacteria and inhibit tissue damage, they also release inflammatory mediators and cause further lung injury [26]. Alveolar macrophages play a major role in LPS-induced neutrophils accumulation in ALI [27]. As neutrophils and alveolar macrophages mediated alveolar damage, pulmonary interstitial edema, and protein leakage-related inflammatory responses, a strategy to limit the lung injury is decreasing the recruitment of these cells in lungs $[15,28]$. Previous studies have demonstrated that ouabain inhibited IL-8 receptors recycling to decrease the migration of neutrophils [29] and decreased intercellular cell adhesion molecule-1 expression in lung tumor cells [30]. Related researches also proposed ouabain reduced the polymorphonuclear leukocytes in animal models of peritonitis induced by concanavalin A and Leishmania amazonensis $[9,10]$. Ouabain was also proven to alleviate allergic airway inflammation in a mouse model, which inhibits inflammatory cell migration into the lungs [11]. Leite and colleagues demonstrated that ouabain reduced neutrophil migration and decreased vascular permeability in peritonitis [12]. In our study, we found ouabain effectively reduced the number of total cells, neutrophils, and macrophages in BALF. We also found ouabain decreased the lung W/D ratio, alleviated the vascular leakage in lungs, and ameliorated proteins in BALF, which indicated that ouabain alleviated the permeability of lungs and reduced serous fluid in lung tissues. Therefore, these results suggested that ouabain could protect against LPS-induced ALI.

NF- $\mathrm{KB}$ signaling pathway plays important roles in the inflammatory responses, which is a pivotal regulator of inflammatory cytokines such as TNF- $\alpha$, IL-1 $\beta$, IL-6, IL-10, and chemokines [31]. LPS can induce p65 dissociating from IKB. Then, p 65 translocated into the nucleus to improve inflammatory cytokines production $[32,33]$. Research indicates that NF-אB signaling pathway could regulate the processes of ALI [34], and inhibiting NF$\kappa B$ signaling could decrease ALI in septic mice [35]. Similar to NF- $\mathrm{KB}$, MAPK family proteins participate in the production of inflammatory cytokines in ALI [17]. In order to investigate the anti-inflammatory effect of ouabain in LPS-induced ALI, we measured the activation of NF-KB and MAPK in lung tissues. We observed the expression of the phosphorylation of ERK, JNK, p38, and p65 in ouabain-treated mice were significantly alleviated, which illustrated the anti-inflammatory effects of ouabain from molecular evidence. Similar results were also observed as researchers reported digitalis, including ouabain, reduced TNF- $\alpha$, IL-1 $\beta$, and IL- 6 levels in LPS-stimulated PBMC by inhibiting NF- $\kappa B$ signaling [36]. A possible mechanism indicated, by interfering with the interaction between tumor necrosis factor receptor 1 and TNFR-associated death domain protein (TRADD) in HeLa cell cultures, ouabain could block the activation of the TNF/NF- $\kappa B$ pathway [8]. In addition, research in pretreatment of ouabain was also proposed as ouabain reduced p65 subunit translocation and IKB degradation in rat hippocampus, thus mediated anti-inflammation and anti-apoptosis [37]. As our results indicated, the MAPK signaling pathway was also regulated by ouabain. The phosphorylated p38 regulates a variety of transcription factors. Ouabain reduces p38 activation in thymocytes after the stimulation of concanavalin A [7], decreases p53 synthesis by inhibiting Src or MAPK activation [38], or decreases the phosphorylation of p38 and NF$\kappa B$ in macrophages [39]. Taken together, with multiple possible targets, ouabain could modulate the function of immune cells in LPS-induced ALI.

The findings in the present work may indicate that ouabain is able to downregulate inflammation in ALI, which may help to discover a new physiological role of ouabain in the immune system. However, there are potential limitations to our current research. First, we revealed the protective effects of ouabain in LPS-induced ALI. However, the underlying molecular mechanisms are poorly understood, and further research is warranted. Second, based on our preliminary experiments, we choose the pretreatment method other than treatment after LPS challenge, which might limit its clinical application in further research.

\section{Conclusions}

Ouabain was able to modulate LPS-induced ALI. It reduced TNF- $\alpha$, IL-1 $\beta$, and IL- 6 levels in lungs, inhibited the number of neutrophils and macrophages, decreased the lung histopathological changes, and ameliorated pulmonary permeability and edema. Further research also has indicated that LPS-induced NF- $\mathrm{KB}$ and MAPK signaling pathways activation is alleviated by ouabain. Taken together, our results provide new evidence for the anti-inflammatory effects of ouabain in vivo. 


\section{Conflict of interests}

None.

\section{Supplementary Figures}
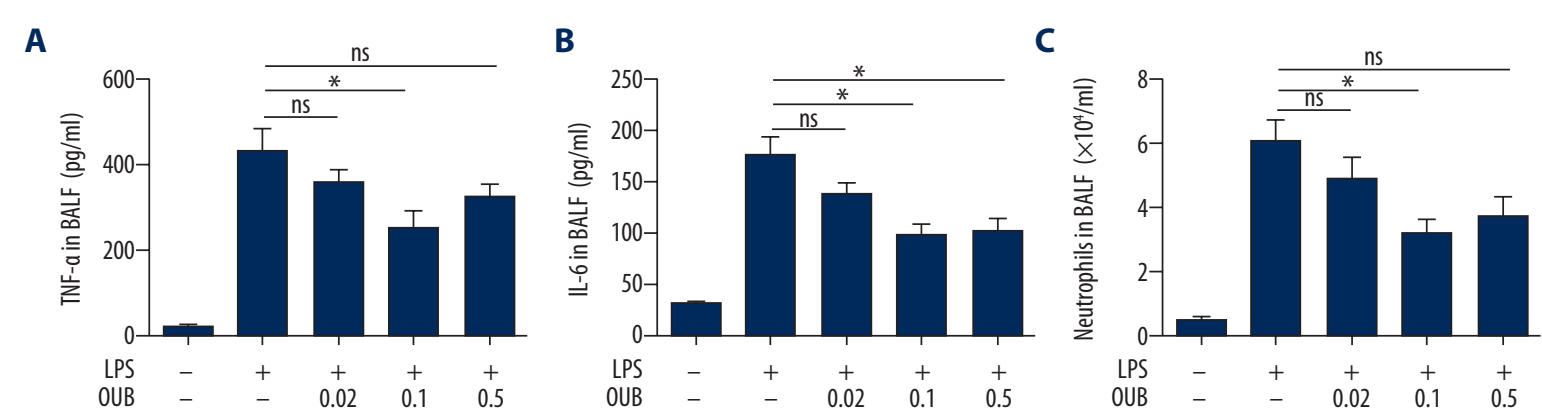

Supplementary Figure 1. The dose-response curve of ouabain administration. Mice received PBS and ouabain $(0.02,0.1,0.5 \mathrm{mg} / \mathrm{kg})$ intraperitoneal injection three consecutive days. One hour after the last injection of the ouabain. Mice were treated with either PBS or LPS ( $5 \mathrm{mg} / \mathrm{kg}$ ) through intranasal instillation for 6 h. (A, B) the levels of cytokines TNF- $\alpha$ and IL- 6 in BALF were detected by ELISA. (C) the infiltration of neutrophils in BALF was measured by flow cytometry. The values presented were mean \pm SEM $(n=6),{ }^{*} P<0.05$.

A

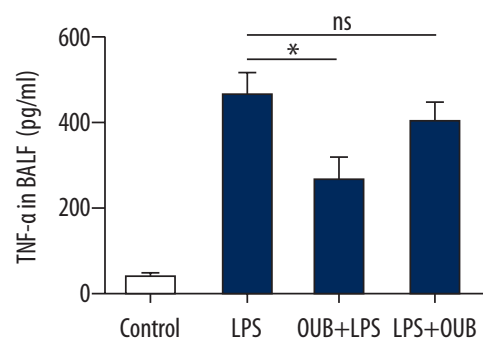

B

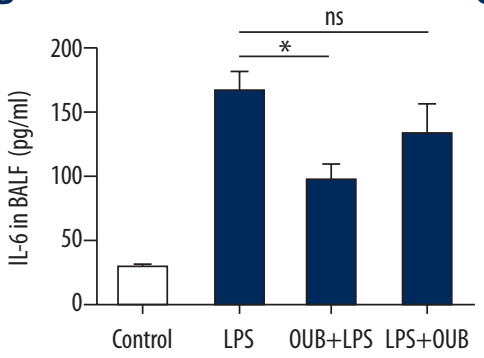

C

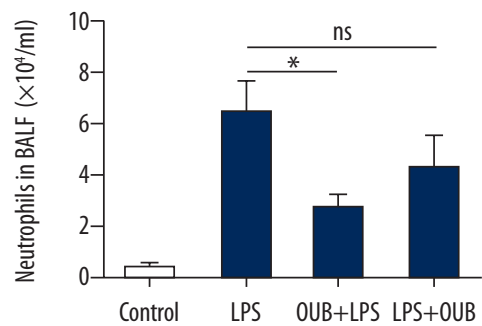

Supplementary Figure 2. Comparison between pre-condition and treatment of ouabain. Mice received ouabain $(0.1 \mathrm{mg} / \mathrm{kg})$ intraperitoneal injection three consecutive days (the group named OUB+LPS) or $1 \mathrm{~h}$ after LPS challenge (the group named LPS+OUB). Mice were treated with either PBS or LPS $(5 \mathrm{mg} / \mathrm{kg}$ ) through intranasal instillation for $6 \mathrm{~h}$. (A, B) the levels of cytokines TNF- $\alpha$ and IL- 6 in BALF were detected by ELISA. (C) the infiltration of neutrophils in BALF was measured by flow cytometry. The values presented were mean $\pm \mathrm{SEM}(\mathrm{n}=6),{ }^{*} P<0.05$.

\section{References:}

1. Han S, Mallampalli RK: The acute respiratory distress syndrome: From mechanism to translation. J Immunol, 2015; 194: 855-60

2. Phua J, Badia JR, Adhikari NK et al: Has mortality from acute respiratory distress syndrome decreased over time?: A systematic review. Am J Respir Crit Care Med, 2009; 179: 220-27

3. Murrell JR, Randall JD, Rosoff J et al: Endogenous ouabain: upregulation of steroidogenic genes in hypertensive hypothalamus but not adrenal. Circulation, 2005; 112: 1301-8

4. el-Masri MA, Clark BJ, Qazzaz HZ, Valdes R: Human adrenal cells in culture produce both ouabain-like and dihydroouabain-like factors. Clin Chem, 2002; 48: 1720-30

5. Blaustein MP, Zhang J, Chen L et al: The pump, the exchanger, and endogenous ouabain: Signaling mechanisms that link salt retention to hypertension. Hypertension, 2009; 53: 291-98
6. Goto A, Yamada K, Nagoshi H et al: Stress-induced elevation of ouabainlike compound in rat plasma and adrenal. Hypertension, 1995; 26: 1173-76

7. Rodrigues-Mascarenhas S, Bloise FF, Moscat J, Rumjanek VM: Ouabain inhibits p38 activation in thymocytes. Cell Biol Int, 2008; 32: 1323-28

8. Yang $Q$, Huang W, Jozwik C et al: Cardiac glycosides inhibit TNF-alpha/NFkappaB signaling by blocking recruitment of TNF receptor-associated death domain to the TNF receptor. Proc Natl Acad Sci USA, 2005; 102: 9631-36

9. Jacob PL, Leite JA, Alves AK et al: Immunomodulatory activity of ouabain in Leishmania leishmania amazonensis-infected Swiss mice. Parasitol Res, 2013; 112: 1313-21

10. de Vasconcelos DI, Leite JA, Carneiro LT et al: Anti-inflammatory and antinociceptive activity of ouabain in mice. Mediators Inflamm, 2011; 2011 912925 
11. Galvão JGFM, Cavalcante-Silva LHA, Carvalho DCM et al: Ouabain attenuates ovalbumin-induced airway inflammation. Inflamm Res, 2017; 66: 1117-30

12. Leite JA, Alves AK, Galvão JG et al: Ouabain modulates zymosan-induced peritonitis in mice. Mediators Inflamm, 2015; 2015: 265798

13. Murata T, Aritake K, Tsubosaka Y et al: Anti-inflammatory role of PGD2 in acute lung inflammation and therapeutic application of its signal enhancement. Proc Natl Acad Sci USA, 2013; 110: 5205-10

14. Xiao Q, Dong N, Yao X et al: Bufexamac ameliorates LPS-induced acute lung injury in mice by targeting LTA4H. Sci Rep, 2016; 6: 25298

15. Dagvadorj J, Shimada K, Chen S et al: Lipopolysaccharide induces alveolar macrophage necrosis via CD14 and the $\mathrm{P} 2 \mathrm{X} 7$ receptor leading to interleukin-1 $\alpha$ release. Immunity, 2015; 42: 640-53

16. Sukriti S, Tauseef M, Yazbeck P, Mehta D: Mechanisms regulating endothelial permeability. Pulm Circ, 2014; 4: 535-51

17. Hu X, Tian Y, Qu S et al: Protective effect of TM6 on LPS-induced acute lung injury in mice. Sci Rep, 2017; 7: 572

18. Nijland R, Hofland T, van Strijp JA: Recognition of LPS by TLR4: Potential for anti-inflammatory therapies. Mar Drugs, 2014; 12: 4260-73

19. Stéphan F, Mabrouk N, Decailliot F et al: Ventilator-associated pneumonia leading to acute lung injury after trauma: Importance of Haemophilus influenzae. Anesthesiology, 2006; 104: 235-41

20. Lee IT, Yang CM: Inflammatory signalings involved in airway and pulmonary diseases. Mediators Inflamm, 2013; 2013: 791231

21. Zhang A, Liu Z, Sheng $L$ et al: Protective effects of syringin against lipopolysaccharide-induced acute lung injury in mice. J Surg Res, 2017; 209: 252-57

22. Teixeira MP, Rumjanek VM: Ouabain affects the expression of activation markers, cytokine production, and endocytosis of human monocytes. Mediators Inflamm, 2014; 2014: 760368

23. Dan C, Jinjun B, Zi-Chun $\mathrm{H}$ et al: Modulation of TNF- $\alpha$ mRNA stability by human antigen $R$ and miR181s in sepsis-induced immunoparalysis. EMBO Mol Med, 2015; 7: 140-57

24. Gonçalves-de-Albuquerque CF, Burth P, Silva AR et al: Murine lung injury caused by Leptospira interrogans glycolipoprotein, a specific $\mathrm{Na} / \mathrm{K}-\mathrm{ATPase}$ inhibitor. Respir Res, 2014; 15: 93

25. Cavalcante-Silva LHA, Lima ÉA, Carvalho DCM et al: Much more than a cardiotonic steroid: Modulation of inflammation by ouabain. Front Physiol, 2017; 8: 895
26. Konrad FM, Reutershan J: CXCR2 in acute lung injury. Mediators Inflamm, 2012; 2012: 740987

27. Harmsen AG: Role of alveolar macrophages in lipopolysaccharide-induced neutrophil accumulation. Infect Immun, 1988; 56: 1858-63

28. Butt Y, Kurdowska A, Allen TC: Acute lung injury: A clinical and molecular review. Arch Pathol Lab Med, 2016; 140: 345-50

29. Ray E, Samanta AK: Receptor-mediated endocytosis of IL-8: A fluorescent microscopic evidence and implication of the process in ligand-induced biological response in human neutrophils. Cytokine, 1997; 9: 587-96

30. Takada Y, Matsuo K, Ogura $\mathrm{H}$ et al: Odoroside A and ouabain inhibit $\mathrm{Na}+/$ $\mathrm{K}+$-ATPase and prevent NF-kappaB-inducible protein expression by blocking $\mathrm{Na}+$-dependent amino acid transport. Biochem Pharmacol, 2009; 78: 1157-66

31. Kawai T, Akira S: Signaling to NF-kappaB by Toll-like receptors. Trends Mol Med, 2007; 13: 460-69

32. Silverman N, Maniatis T: NF-kappaB signaling pathways in mammalian and insect innate immunity. Genes Dev, 12001; 5: 2321-42

33. Hoffmann A, Levchenko A, Scott ML, Baltimore D: The IkappaB-NF-kappaB signaling module: Temporal control and selective gene activation. Science, 2002; 298: 1241-45

34. Imai Y, Kuba K, Neely GG et al: Identification of oxidative stress and Tolllike receptor 4 signaling as a key pathway of acute lung injury. Cell, 2008; 133: 235-49

35. Shen W, Gan J, Xu S et al: Penehyclidine hydrochloride attenuates LPSinduced acute lung injury involvement of NF-kappaB pathway. Pharmacol Res, 2009; 60: 296-302

36. Heese K, Fiebich BL, Bauer J, Otten U: NF-kappaB modulates lipopolysaccharide-induced microglial nerve growth factor expression. Glia, 1998; 22: 401-7

37. Kinoshita PF, Yshii LM, Vasconcelos AR et al: Signaling function of Na,KATPase induced by ouabain against LPS as an inflammation model in hippocampus. J Neuroinflammation, 2014; 11: 218

38. Laflamme N, Soucy G, Rivest S: Circulating cell wall components derived from gram-negative, not gram-positive, bacteria cause a profound induc tion of the gene-encoding Toll-like receptor 2 in the CNS. J Neurochem, 2001; 79: 648-57

39. Alves SMLG: Effect of ouabain on NFKB and p-38 activation in macrophages BMC Proc, 2014; 8(Suppl. 4): p260 